Pesq. Vet. Bras. 35(11):925-932, novembro 2015 DOI: 10.1590/S0100-736X2015001100009

\title{
Características do sêmen a fresco e descongelado de garanhões da raça Nordestina ${ }^{1}$
}

\author{
Maria Alice M. Santos², Adriana Gradela ${ }^{3 *}$, Elenice A. Moraes ${ }^{2}$, Wildelfrancys L. \\ Souza $^{2}$, Nadja G. Alves ${ }^{4}$, Jonathan M. Silva Costa $^{2}$ e Wasley C.G. Matos ${ }^{2}$
}

\begin{abstract}
Santos M.A.M., Gradela A., Moraes E.A., Souza W.L., Alves N.G., Costa J.M.S. \& Matos W.C.G. 2015. [Sperm characteristics in fresh and cryopreserved semen of Nordestino stallions breed.] Características do sêmen a fresco e descongelado de garanhões da raça Nordestina. Pesquisa Veterinária Brasileira 35(11):925-932. Colegiado de Medicina Veterinária, Fundação Universidade Federal do Vale do São Francisco, Rodovia $407 \mathrm{Km}$ 12, Lote 543, Projeto Nilo Coelho C1, Petrolina, PE 56300-000, Brazil. E-mail: agradela@hotmail.com

This paper describes the seminal characteristics of the plasma membrane of frozenthawed sperm. Nineteen ejaculates of Nordestino horse breed. The aspects analyzed in the physical parameters of fresh semen were total and progressive motility and your longevity after dilution or thawed; sperm morphology, functional and structural integrity of the plasma membrane of the sperm and the sperm-binding ability to the perivitelline membrane of the yolk (MPV) after thawed. The variables were assessed by ANOVA with post hoc test of Student Newman-Keuls test $(\mathrm{P}<0.05)$. The total and progressive motility were higher in diluted semen than thawed $(\mathrm{P}<0.05)$. The average percentage of the major, minor and total defects was lower than the limit recommended by the CBRA. The percentage of reactive to hypo-osmotic swelling test was $14.21 \pm 1.12 \%$, the intact membrane detected by supravitally test was $62.22 \pm 9.06 \%$ and the SYBR-14 was $81.47 \pm 26.9$. The ability of sperm to bind to the MPV after thawing semen was $230.39 \pm 57.09$. The total and progressive motility at time 0 min of termo resistance test was higher than to 150 minutes $(\mathrm{P}<0.05)$, and no difference was observed in the times 10 and 30 minutes. The results demonstrate that the use of additional laboratory tests help in the process of evaluation of samples, making possible to obtain more reliable and accurate information. Although cryopreservation has caused decrease in sperm motility and was used diluents with amides to diluted and cryopreservation protocol and this minimized the osmotic damage to sperm cells and maintained the morphological, functional and structural integrity of the plasma membrane of the sperm. These results are a reference for future studies since there are no comparative data on this breed.
\end{abstract}

INDEX TERMS: CASA, total motility, progressive motility, SYBR-14, hypoosmotic test.

RESUMO.- Este estudo descreveu as características seminais, da membrana plasmática e do acrossoma de espermatozoide congelado/descongelado de 19 ejaculados de garanhões da raça Nordestina. Os aspectos analisados incluíram

\footnotetext{
${ }^{1}$ Recebido em 12 de janeiro de 2015.

Aceito para publicação em 22 de setembro de 2015.

${ }^{2}$ Programa de Pós-Graduação em Ciência Animal, Universidade Federal do Vale do São Francisco (Univasf) Rodovia $407 \mathrm{Km} \mathrm{12}$, Lote 543, Projeto Nilo Coelho C1, Petrolina, PE 56300-000, Brasil.

${ }^{3}$ Colegiado de Medicina Veterinária, Univasf, Petrolina, PE 56300-000. *Autor para correspondência: agradela@hotmail.com

${ }^{4}$ Departamento de Zootecnia, Universidade Federal de Lavras (UFLA), Cx. Postal 3037, Av. Doutor Sylvio Menicucci 1001, Lavras, MG 37200-000.
}

os parâmetros físicos do sêmen fresco; a motilidade e a longevidade do sêmen diluído e descongelado; a morfologia espermática, integridade funcional e estrutural da membrana plasmática do espermatozoide e a habilidade de ligação do espermatozoide à membrana perivitelina da gema do ovo de galinha do sêmen descongelado. As variáveis foram avaliadas pela ANOVA com post hoc teste de Student Newman-Keuls $(\mathrm{P}<0,05)$. A MT e a MP foram maiores $(\mathrm{P}<0,05)$ no sêmen diluído do que no descongelado. A percentagem média de defeitos maiores, menores e totais foi muito inferior ao limite recomendado pelo CBRA. A porcentagem de reativos ao HOST foi de $14,21 \pm 1,12 \%$ e a porcentagem média de membranas íntegras detectadas pelo teste supravital 
de $62,22 \pm 9,06 \%$ e pela sonda SYBR-14 de $81,47 \pm 26,90$. 0 número médio de espermatozoides ligados à MPV após a descongelação do sêmen foi de 230,39 57,09 . A MT e MP no tempo 0 min do TTR foi superior $(\mathrm{P}<0,05)$ em relação a 150 min, não diferindo nos tempos $10 \mathrm{~min}$ e $30 \mathrm{~min}$. Os resultados demonstram que a utilização dos testes laboratoriais adicionais ajudam no processo de avaliação das amostras, possibilitando a obtenção de informações mais confiáveis e precisas. Embora a criopreservação tenha provocado queda na motilidade seminal, o uso de diluidor contendo amidas minimizou os danos osmóticos nas células espermáticas e manteve a integridade morfológica, funcional e estrutural da membrana plasmática do espermatozoide. Estes resultados são um referencial em estudos futuros uma vez que, inexistem dados comparativos nesta raça.

TERMOS DE INDEXAÇÃO: CASA, motilidade total, motilidade progressiva, SYBR-14, teste hiposmótico.

\section{INTRODUÇÃO}

0 rebanho equídeo brasileiro é de 7.793 milhões de animais (IBGE 2010), dos quais 7,5 milhões são equinos (CNA 2006). Como parte deste efetivo, encontra-se o cavalo Nordestino, o qual é uma das maiores riquezas do Nordeste brasileiro (Melo 2011).

A raça Nordestina é descendente do cavalo Barbo-árabe, originário de Portugal e Espanha e introduzido no Brasil no período colonial, ao qual se assemelha morfologicamente (Costa et al. 1974). A altura mínima padrão é de cerca de 1,30m para machos, sendo a ideal de 1,38m a $1,46 \mathrm{~m}$, e $1,27 \mathrm{~m}$ para as fêmeas, ideal de $1,35 \mathrm{~m}$ a $1,43 \mathrm{~m}$ (ABCCN 1987); o peso por volta de $350 \mathrm{~kg}$ e as pelagens predominantes a tordilha e a castanha (Torres \& Jardim 1992). Esta raça se caracteriza pela rusticidade, sobriedade, rigidez muscular, temperamento sociável e amistoso, fácil adaptação ao meio e ao processo de criação, sendo utilizada principalmente no Nordeste brasileiro para o trabalho com o gado, pois podem sobreviver com pouco alimento e água, viajando grandes distâncias. É, portanto, essencial em seu ambiente natural, a caatinga agressiva, onde presta relevantes serviços à economia da região, não só transportando cargas, como também pessoas (Enciclopédia 2014). Entretanto, com o fechamento da Associação Brasileira dos Criadores do Cavalo Nordestino (ABCCN) no início da década de 90, muitos desses animais passaram a se reproduzir ao acaso. As fêmeas têm sido acasaladas com reprodutores Mangalarga Marchador e Quarto-de-Milha para produção de mestiços para vaquejadas (Costa et al. 2001) e os melhores machos castrados e utilizados na lida do gado. Portanto, é imprescindível a realização de programas que promovam a seleção, preservação e reprodução desse genoma, principalmente, em virtude do risco de extinção da raça e das transformações climáticas do planeta.

Dentre as biotécnicas utilizadas na reprodução animal para promover o melhoramento genético, destaca-se a inseminação artificial (IA), que aliada à criopreservação de sêmen representa uma importante ferramenta na preservação da raça equina, tanto pela maximização do uso de reprodutores superiores quanto pela formação de um banco genético disponível por tempo indeterminado (Samper \& Hankins 2001). Embora apresente inúmeras vantagens, a criopreservação apresenta limitações em várias raças equinas (Alvarenga et al. 2004), que geram um entrave à sua disseminação (Brinsko et al. 2000, Batellier et al. 2001, Carmo \& Almeida 2006). Isto porque o congelamento/descongelamento do sêmen ocasiona prejuízos, muitas vezes irreversíveis, aos espermatozoides como perda de motilidade, alterações estruturais e funcionais da membrana espermática e, consequentemente, diminuição do poder fecundante. Por isso o uso de testes na avaliação das injúrias é fundamental para que se produza um protocolo de congelamento/descongelamento ideal.

0 potencial de fertilização do espermatozoide depende da integridade física e funcional de sua membrana plasmática (Oliveira 2007), sendo difícil que um único teste laboratorial seja capaz de determinar a fertilidade seminal (Garcia 2005). Por isto ao se avaliar um sêmen devemos lançar mão de diversas técnicas para avaliar as características físicas do ejaculado, a motilidade e longevidade dos espermatozoides, a funcionalidade e integridade estrutural das membranas espermáticas. Por exemplo, a motilidade espermática constitui-se num recurso muito utilizado para avaliação dos efeitos da criopreservação sob os espermatozoides e para se obter comparações entre os diluentes, métodos de resfriamento e congelação e diluições utilizadas (Kenney et al. 1983, Varner et al. 1988, Pickett \& Amann 1993), pois é um elemento indispensável para a fecundação (Oliveira 2007).

Outro parâmetro essencial é a avaliação morfológica, uma vez que, deformidades na célula espermática podem conferir diminuição na capacidade de fertilização (Kavak et al. 2004, Pugliesi 2009). Embora a morfologia espermática dos equídeos se assemelhe as demais espécies, a cabeça do espermatozoide apresenta formato assimétrico, o acrossoma é relativamente pouco desenvolvido e a inserção abaxial da cauda é considerada normal (Canisso et al. 2008). A avaliação andrológica classifica as anormalidades espermáticas subdividindo-as em defeitos maiores e defeitos menores (CBRA 2013). A funcionalidade das membranas espermáticas pode ser avaliada através do teste hiposmótico (HOST) (Linden 2012), que permite observar o comportamento do espermatozoide, com membrana celular íntegra, quando submetido à solução hiposmótica. Assim, o influxo de água para o interior das células causa aumento de volume celular (edema) e, consequentemente, dobramento da cauda em espermatozoides que possuem a membrana funcionalmente intacta (Chapon 2011).

Por outro lado, a integridade estrutural da membrana plasmática do espermatozoide pode ser avaliada pelo teste supravital, que utiliza corantes derivados da fluoresceína como a eosina e, como corante de fundo, a nigrosina. Assim, a eosina ao penetrar pela membrana lesionada dos espermatozoides cora o interior do núcleo em vermelho, identificando os espermatozoides mortos. É considerada uma técnica rápida, de fácil manipulação o que dispensa o uso de microscópio especial (Ker 2010). As técnicas que utilizam corantes fluorescentes (sondas epifluorescentes ou fluorocromos) estão se tornando cada vez mais importantes para 
a avaliação da integridade da membrana plasmatica do esperatozoide (Pereira et al. 2012). Dentre as sondas, o iodeto de propídio cora em vermelho o núcleo de células com membrana plasmática lesada (Arruda 2000, Arruda et al. 2003, Celeghini 2005), enquanto que a SYBR-14 atravessa a membrana plasmática íntegra corando o núcleo dos espermatozoides em verde (Pereira et al. 2012).

A capacidade fecundante da célula espermática pode ser avaliada pelo teste de ligação do espermatozoide ao ovócito, de forma que o teste de penetração in vitro (PIV) em ovócitos simula os processos envolvidos no mecanismo de fertilização (Bernardi 2008). As similaridades entre as glicoproteínas da zona pelúcida e da membrana perivitelina (MPV) do ovo de galinha, permitem a ligação dos espermatozoides de outras espécies a esta MPV (Mugnier et al. 2009) e simula a interação dos gametas (Corcini 2010) sendo, portanto, um teste eficiente para se verificar a habilidade dos espermatozoides em sofrer a reação acrossomal (Corcini et al. 2012). 0 uso da MPV do ovo de galinha, em relação aos ovócitos, é muito mais vantajoso porque permite, sobre diferentes fragmentos de uma mesma membrana, analisar diferentes partidas de sêmen, minimizando o efeito fêmea na análise e também por ser menos dispendioso que o ovócito (Csermak Junior 2011). Por isto é considerado um valioso teste na análise da eficácia do processo de criopreservação do sêmen (Barbato et al. 1998).

A longevidade dos espermatozoides diluídos e descongelados pode ser avaliada através do teste de termo resistência (TTR), que avalia a motilidade e o vigor espermáticos no tempo zero (momento quando o sêmen é descongelado) e em períodos de tempo que variam de 30 a 90, 180 e 240 minutos (Furst 2006, Oliveira 2010). Este teste pode ser afetado pelo efeito do ejaculado, método de congelamento e efeitos do tempo incubação (Aidar 2013).

A despeito dos conhecimentos já alcançados, ainda não há padronização de um protocolo para congelação de sêmen equino, sendo comum a adoção de protocolos individualizados para cada garanhão (Loomis \& Graham 2008). Isto se torna particularmente necessário quando se fala da raça Nordestina, cujas características seminais e resposta à criopreservação são ainda desconhecidas.

O presente estudo objetivou analisar as características do sêmen à fresco e descongelado de garanhões da raça Nordestina. Para tanto se avaliou os parâmetros físicos do sêmen fresco; a motilidade e a longevidade do sêmen diluído e descongelado e a morfologia espermática, integridade funcional e estrutural da membrana plasmática do espermatozoide e a habilidade de ligação do espermatozoide à membrana perivitelina da gema do ovo de galinha do sêmen descongelado.

\section{MATERIAL E MÉTODOS}

Foram selecionados dois garanhões da raça Nordestina, com média de 9,5 anos de idade, mantidos em baias individuais, clinicamente sadios e com histórico de fertilidade normal, provenientes da fazenda da Associação Brasileira de Criadores do Cavalo Nordestino, localizada em Juazeiro, BA $\left(09^{\circ} 25^{\prime} 00^{\prime \prime}\right.$ de latitude S; $40^{\circ} 30^{\prime} 00^{\prime \prime}$ de longitude 0). Este estudo foi aprovado pelo Comitê de Ética Experimental em Humanos e Animais (CEEHA) da Univasf sob protocolo $\mathrm{n}$ o $0006 / 161012$.

Antes do período experimental, os garanhões foram submetidos ao esgotamento das reservas espermáticas extragonadais realizando-se três coletas seminais diárias, por oito dias, utilizando-se vagina artificial modelo Hannover ${ }^{\circledR}$ (Biotech, Botucatu/SP, Brasil) e uma fêmea em estro natural ou induzido como manequim. Os animais receberam ração industrializada (Durancho ${ }^{\circledR}$ 12MA); capim bermuda vaqueiro (Cynodon dactylon) e capim-cameron (Pennisetum purpureum) picados e sal mineral e água ad libitum, segundo as exigências nutricionais da categoria.

Protocolo de coleta e manipulação do sêmen. Dois dias após a última coleta do esgotamento, o sêmen de cada um dos animais começou a ser coletado com vagina artificial e fêmea em estro para análise, realizando-se três coletas por semana, com intervalos de 48 horas entre cada uma. Foram obtidos 19 ejaculados ( $\mathrm{N}=10$ do animal 01 e $\mathrm{N}=9$ do animal 2).

Após a coleta, o ejaculado era filtrado com auxílio de um filtro de nylon e o volume determinado por visualização direta em tubo falcon graduado. Em seguida, efetuavam-se as análises físicas do sêmen, como aspecto (aquoso, leitoso e cremoso), coloração (branca, branca-amarelada e amarelada) e odor e, sob microscopia convencional, a motilidade individual progressiva (0-100\%) e vigor (0-5) de acordo com CBRA (2013). Após diluição (1:1) com diluidor de transporte (BotuSêmen ${ }^{\circledR}$, Biotech Botucatu, Botucatu, $\mathrm{SP}$, Brasil) pré-aquecido a $37^{\circ} \mathrm{C}$, o sêmen foi homogeneizado e encaminhado para o laboratório do Centro de Pesquisa em Suínos, Espécies Nativas e Silvestres (CPSENS) da Univasf, localizado no Campus de Ciências Agrárias em Petrolina, PE, protegido da luz.

No CPSENS o sêmen foi submetido à centrifugação em tubos cônicos $(50,0 \mathrm{~mL})$ a $2200 \mathrm{rpm}$ por 15 minutos, o sobrenadante descartado e a concentração espermática determinada utilizando um espectrofotômetro (Spermacue ${ }^{\circledR}$, Minitub, Berlin, Alemanha). Para isto, utilizou-se uma curveta contendo 3,0 mL de água destilada e $120 \mu \mathrm{L}$. Os peletes foram ressuspendidos no diluidor de congelação (BotuCrio ${ }^{\circledR}$, Biotech Botucatu, Botucatu, SP, Brasil), pré-aquecido a $37^{\circ} \mathrm{C}$, até a concentração de $10^{6}$ espermatozoides por $\mathrm{mL}$ e avaliados quanto às motilidades total (MT) e progressiva (MP) no CASA. Ejaculados com pelo menos 70,0\% de MT foram submetidos à congelação (CBRA 2013).

As amostras seminais foram envasadas em palhetas de 0,5 $\mathrm{mL}$ de polietileno, lacradas com seladora ultrasônica de palhetas (UltraSeal ${ }^{\circledR}$ Minitub, Berlin, Alemanha) e estabilizadas em câmara fria (Gemar ${ }^{\circledR}$, Gefrio, Fortaleza, CE, Brasil) a $5^{\circ} \mathrm{C}$, por 20 minutos. Após, foram submetidas ao resfriamento rápido em vapor de nitrogênio $\left(-120^{\circ} \mathrm{C}\right)$ por 15 minutos, também dentro da câmara fria e, posteriormente, imersas em nitrogênio líquido e, então, armazenadas à $-196^{\circ} \mathrm{C}$, para posterior análise.

As palhetas foram descongeladas em banho-maria a $37^{\circ} \mathrm{C}$, por trinta segundos (Dell'Aqua Junior 2000) e submetidas às análises.

Avaliação da Motilidade Total (MT) e Progressiva (MP). A avaliação da Motilidade Total (MT) e Progressiva (MP) foi realizada no sistema de análise espermática computadorizada (CASA ${ }^{\circledR}$; MINITUB, Berlin, Alemanha) utilizando-se o software (Spermvision ${ }^{\circledR}$; Minitub, Berlin, Alemanha). Amostras de 8,0 $\mu \mathrm{L}$ de cada amostra de sêmen diluído e descongelado foram colocados entre lâmina e lamínula, pré-aquecidas a $37^{\circ} \mathrm{C}$, e avaliadas quanto às motilidades total (MT, \%) e progressiva (MP, \%).

Avaliação da morfologia espermática. A morfologa espermática foi avaliada no sêmen descongelado sob microscópio de luz com contraste de fase (200x) através da técnica de preparação úmida. Para tanto uma alíquota de $10 \mu \mathrm{L}$ de sêmen foi adicionada a $1,0 \mathrm{~mL}$ de formol-salino tamponado, aquecido a $37^{\circ} \mathrm{C}$, e estocada à temperatura ambiente até a análise. Foram feitas lâminas pelo método da câmara úmida e contadas 200 células no aumento de 
1000x. Os defeitos espermáticos totais foram subdivididos em defeitos maiores e menores (CBRA 2013).

Avaliação da integridade funcional da membrana plasmática dos espermatozoides descongelados. Para avaliar a integridade funcional da membrana plasmática do espermatozoide utilizou-se o teste hiposmótico (HOST) em câmara úmida. Uma amostra de $100 \mu \mathrm{L}$ de sêmen descongelado diluído em $1,0 \mathrm{~mL}$ de solução à base de citrato de sódio e frutose a $125 \mathrm{mOsm} / \mathrm{L}$ foi incubado em banho-maria a $37^{\circ} \mathrm{C}$ por 30 minutos (Melo et al. 2005). Após esse período, $15 \mu \mathrm{L}$ dessa solução foram avaliados em câmara úmida, sob microscopia de contraste de fase, em aumento de 400x. Foram contadas 100 células, considerando-se a presença ou ausência de cauda enrolada (Neild et al. 2000). 0 cálculo da reação da reação hiposmótica foi realizado pela fórmula: HOST= (\% de caudas enroladas após o HOST) - (\% de caudas enroladas antes do HOST) conforme Melo \& Henry (1999).

Avaliação da integridade estrutural da membrana plasmática dos espermatozoides descongelados. 0 teste supravital (vivos e mortos) foi utilizado para avaliar a integridade estrutural da membrana plasmática do espermatozoide, utilizando-se o corante eosina - nigrosina a $0,5 \%$. Amostras de $10 \mu \mathrm{L}$ de sêmen foram incubadas com $10 \mu \mathrm{L}$ de corante sobre lâmina de vidro aquecida a $37^{\circ} \mathrm{C}$ por $3 \mathrm{~min}$. Após incubação confeccionou-se um esfregaço para realização da leitura, sob microscopia óptica comum, em aumento de 400x. Foram contados 100 espermatozoides e classificados como vivos os que não tiveram o núcleo corado de vermelho (Dott \& Foster 1972).

Avaliação da integridade da membrana plasmática dos espermatozoides descongelados. A integridade da membrana plasmática dos espermatozoides descongelados foi avaliada pelo teste com sonda fluorescente. Para esta avaliação utilizou-se a associação de duas sondas fluorescentes, o iodeto de propídio e o SYBR-14. Uma amostra de 0,5mL de sêmen descongelado foi colocada em tubos plásticos de 1,5mL juntamente com $0,5 \mathrm{~mL}$ de STALP, $5 \mu \mathrm{L}$ de SYBR-14 (solução de $20 \mu \mathrm{M}$ em $\mathrm{Me}_{2} \mathrm{SO}$ ) e $10 \mu \mathrm{L}$ de iodeto de propídio (solução de 2,4mM em água; Molecular Probes, Eugene OR) como descrito por Garner et al. (1994). A amostra foi incubada à temperatura ambiente por 8 minutos ao abrigo de luz e uma alíquota de $6,5 \mu \mathrm{L}$ da solução foi utilizada para preparo da câmara úmida, entre lâmina e lamínula, pré-aquecida a $37^{\circ} \mathrm{C}$. A leitura foi realizada em microscopia de fluorescência (Axio Imager 2, ZEISS ${ }^{\circledR}$, Berlin, Alemanha). Nas leituras identificaram-se um mínimo de 200 células por amostra e o padrão de emissão de fluorescência foi: membrana plasmática lesada - vermelho fluorescente (Iodeto de propídio); membrana plasmática intacta - verde fluorescente (SYBR-14).

Avaliação da habilidade do espermatozoide equino se ligar a membrana de ovócitos. A habilidade do espermatozoide equino se ligar a membrana de ovócitos foi avaliada utilizando-se a membrana perivitelina (MPV) da gema de ovo de galinha (Moraes et al. 2010). A MPV foi preparada pela separação da gema da clara do ovo, com a remoção do excesso de clara utilizando um papel-filtro e a gema intacta colocada sobre um pedaço de parafilme. Sua membrana foi delicadamente rompida e lavada várias vezes com STALP e, então, espalhada delicadamente em uma placa petri e cortada em pequenos quadrados $(1 \mathrm{x} 1 \mathrm{~cm})$ utilizando-se uma cubeta de espectrofotômetro como modelo. Cada pedaço de membrana foi colocado em um tubo de cultura $(16 \times 100 \mathrm{~mm})$ contendo $1 \mathrm{~mL}$ de meio de incubação (STALP) e incubado com 50.000 espermatozoides por 2 horas a $37^{\circ} \mathrm{C}$ numa atmosfera de $5 \%$ de $\mathrm{CO}_{2}$. A cada 30 minutos, os tubos foram agitados para manter a membrana aberta. Meia hora antes do final da incubação, acrescentou-se $1 \mu \mathrm{L}$ de Hoechst 33342 (1mg/mL de água) para corar o núcleo dos espermatozoides. Depois da incubação, cada membrana foi colocada em um tubo contendo 1,0mL de STALP e as membranas foram lavadas cinco vezes para remoção dos espermatozoides que não se ligaram. Na sequência, cada membrana colocada sobre uma lâmina foi gentilmente aberta, coberta com lamínula e analisada utilizando-se microscópio de fluorescência (Axio Imager 2, ZEISS ${ }^{\circledR}$, Berlim, Alemanha). 0 número de espermatozoides ligados à membrana foi determinado contando-se seis campos aleatórios de cada membrana, e o número médio de espermatozoides ligados por membrana calculado.

Avaliação da longevidade dos espermatozoides diluídos e descongelados. A longevidade dos espermatozoides diluídos e descongelados foi avaliada pelo teste de termo resistência (TTR), que consistiu no acondicionamento de $1,0 \mathrm{~mL}$ da amostra de sêmen em tubos plásticos de $1,5 \mathrm{~mL}$ em banho-maria a $37^{\circ} \mathrm{C}$ por 150 minutos. Foram realizadas avaliações da MT e MP no CASA, sendo as leituras realizadas nos tempos 0, 10, 30 e 150 minutos de incubação.

Análise Estatística. Os dados foram comparados pela ANOVA com post hoc Teste de Student Newman Keul $(\mathrm{P}<0,05)$ utilizando o SAEG (UFV 1997). Correlação simples foi testada entre as variáveis (motilidade espermática total e progressiva, reativos ao HOST, íntegros pelo teste de fluorescência, não corados pelo teste supravital e ligados à MPV do ovo de galinha), para verificar possíveis relações entre as características estudadas.

\section{RESULTADOS}

O presente estudo avaliou as características do sêmen a fresco e descongelado de dezenove ejaculados de dois garanhões da raça Nordestina.

No Quadro 1 observa-se, em valores de média \pm SD, o resultado das avaliações físico-morfológicas e funcionais do sêmen diluído e descongelado de garanhões da raça Nordestina. A MT do sêmen diluído avaliada no CASA foi maior $(\mathrm{P}<0,05)$ que a do sêmen descongelado. 0 mesmo ocorreu com a MP. 0 principal defeito maior observado foi cauda fortemente dobrada $(4,14 \pm 2,31 \%)$, seguida pela gota citoplasmática proximal $(2,58 \pm 1,63 \%)$, cabeça isolada $(1,69 \pm 1,49 \%)$, acrossoma incompleto $(1,31 \pm 1,19 \%)$ e cauda dobrada com gota $(0,89 \pm 0,82 \%)$. 0 principal defeito menor observado foi cauda dobrada simples $(5,14 \pm 1,93 \%)$, seguida de cabeça delgada $(4,58 \pm 2,09 \%)$, cabeça alongada $(3,86 \pm 1,78 \%)$ e gota citoplasmática distal $(1,39 \pm 1,34 \%)$. Os valores percentuais da integridade funcional espermática do sêmen imediatamente pós-descongelamento $(0 \mathrm{mOsm})$ e após o HOST $(125 \mathrm{mOsm})$ não diferiram $(\mathrm{P}>0,05)$ tendo sido, respectivamente, $40,61 \pm 7,97 \%$ e $54,82 \pm 9,09 \%$, de modo que a porcentagem de reativos ao HOST foi de $14,21 \pm 1,12 \%$. A porcentagem de células espermáticas íntegras $(62,22 \pm 9,06 \%)$ no sêmen descongelado avaliada pelo teste supravital foi menor $(\mathrm{P}<0,05)$ que a porcentagem de células íntegras avaliadas pela sonda SYBR-14 e o número médio de espermatozoides ligados à MPV após a descongelação do sêmen foi de 230,39 $\pm 57,09$.

As médias de MT e MP, tanto do sêmen diluído quanto do pós-descongelado, submetidos ao TTR estão exibidas no Quadro 2. Observou-se que, tanto a MT quanto a MP no tempo 0 foram superiores às observadas no tempo de 150 min $(\mathrm{P}<0,05)$. Houve pronunciada $(\mathrm{P}<0,05)$ queda da MT e MP do sêmen descongelado em comparação ao sêmen diluído no tempo 0 min do TTR $(41,05 \%$ e 41,52\%, respectivamente), assim como aos $150 \mathrm{~min}$ do TTR $(40,34 \%$ e $33,56 \%$ ). 
Quadro 1. Média + SD das características físico-morfológicas e funcionais do sêmen diluído e descongelado de garanhões da raça Nordestina

\begin{tabular}{lccc}
\hline & \multicolumn{3}{c}{ Sêmen } \\
\cline { 2 - 4 } & Fresco (MC) & Diluído (CASA) & Descong. (CASA) \\
\hline Características Físicas & & & - \\
Volume (mL) & $29,83 \pm 12,60$ & - & - \\
Aspecto & Leitoso & - & - \\
Coloração & branca-acinzentada & - & - \\
Odor & Sui generis & - & $34,08 \pm 12,57 \mathrm{~B}$ \\
Motilidade Total (\%) & $80,91 \pm 9,29$ & $72,15 \pm 9,34 \mathrm{~A}$ & $30,53 \pm 12,82 \mathrm{~B}$ \\
Motilidade Progressiva (\%) & - & $59,24 \pm 11,45 \mathrm{~A}$ & 20, \\
Vigor (0-5) & 3 & - & $2,12 \pm 1,93$ \\
Características Morfológicas & - & - & $3,74 \pm 2,29$ \\
\%Defeitos Maiores & - & - & $5,86 \pm 4,22$ \\
\%Defeitos Menores & - & - & $14,21 \pm 1,12$ \\
\%Defeitos Totais & & - & $62,22 \pm 9,06 \mathrm{a}$ \\
Características Funcionais & - & - & $81,47 \pm 26,90 \mathrm{~b}$ \\
\% Sptzs reativos ao HOST & - & - & $230,39 \pm 57,09$ \\
\% Sptz com MP íntegra pelo teste supravital & - & &
\end{tabular}

MC = Microscopia Convencional, Descong.= Descongelado. A,B Valores de Média + desvio padrão na mesma linha, a,b Valores de Média + desvio padrão na mesma coluna diferem entre sí $(\mathrm{P}<0,05)$ pelo teste de Student Newman-Keuls.

Quadro 2. Médias de motilidade espermática total e progressiva do sêmen diluído e descongelado de garanhões da raça Nordestina após o teste de termo resistência (TTR)

\begin{tabular}{lcccr}
\hline Motilidade & \multicolumn{4}{c}{ Sêmen diluído } \\
\cline { 2 - 5 } & 0 & 10 & 30 & 150 \\
\hline Total & $71,29 \pm 2,11 \mathrm{~A}$ & $68,41 \pm 2,57 \mathrm{AB}$ & $66,20 \pm 2,64 \mathrm{AB}$ & $54,52 \pm 5,01 \mathrm{~B}$ \\
Progressiva & $58,62 \pm 2,99 \mathrm{~A}$ & $55,10 \pm 2,96 \mathrm{~A}$ & $52,24 \pm 3,50 \mathrm{~A}$ & $39,00 \pm 5,22 \mathrm{~B}$ \\
& \multicolumn{4}{c}{ Sêmen descongelado } \\
Total & $30,24 \pm 3,07 \mathrm{~A}$ & $26,10 \pm 3,30 \mathrm{AB}$ & $23,64 \pm 3,13 \mathrm{AB}$ & $14,18 \pm 2,90 \mathrm{~B}$ \\
Progressiva & $17,10 \pm 2,83 \mathrm{~A}$ & $12,88 \pm 2,78 \mathrm{AB}$ & $11,83 \pm 3,02 \mathrm{AB}$ & $5,44 \pm 1,53 \mathrm{~B}$
\end{tabular}

Correlação positiva muito alta $(\mathrm{P}<0,01)$ foi observada entre motilidade total e motilidade progressiva tanto para o sêmen diluído $(\mathrm{r}=0,95)$ quanto para o sêmen descongelado $(r=0,88)$.

\section{DISCUSSÃo}

O congelamento/descongelamento do sêmen ocasiona prejuízos, muitas vezes irreversíveis, aos espermatozoides como a perda de motilidade e alterações estruturais e funcionais da membrana espermática, que causam diminuição da fertilidade. Os métodos convencionais (motilidade, vigor e morfologia espermática) de avaliação da qualidade seminal apresentam limitada capacidade para identificar diferenças de menor magnitude entre machos com potencial de fertilidade mais homogênea (Gadea 2005), tornando os métodos in vitro mais eficientes no diagnóstico do potencial fertilizante dos machos (Corcini 2010).

Neste estudo o volume médio e as características físicas do sêmen fresco estavam dentro dos valores e padrões considerados normais para equinos pelo CBRA (2013). O volume médio foi superior ao descrito para Quarto de Milha e Mangalarga Marchador por Costa et al. (2014) e inferior aos de jumento Pêga por Oliveira (2010) e Mangalarga Marchador por Candeias (2010). 0 mesmo foi observado com a motilidade total e o vigor espermáticos (motilidade
$>70,0 \%$ e vigor $\geq 3$ ), cujos valores foram superiores aos de observados por Costa et al. (2014) e semelhantes aos de Candeias (2010) e Oliveira (2010).

A avaliação de múltiplas características espermáticas em uma única amostra de sêmen com alto grau de acurácia e repetibilidade pode ser realizada através do sistema automatizado de análise de motilidade (CASA) (Mortimer \& Maxwell 2004), que tem a vantagem de diminuir a ocorrência de erros e, por sua objetividade, permitir avaliações adicionais, tais como a velocidade e o tipo de trajetória dos espermatozoides. Contudo, sua grande desvantagem são os altos custos de cada avaliação (Aurich 2005). Neste estudo a queda na motilidade total do sêmen descongelado foi inferior à observada em Quarto de Milha e Mangalarga Marchador, enquanto que a queda na motilidade progressiva foi inferior a de Quarto de Milha e superior à de Mangalarga Marchador (Costa et al. 2014). Medeiros et al. (2002) comparando crioprotetores com diferentes concentrações de amida (3 a 5\%) e a base de glicerol observaram motilidade total de 49 a $52 \%$ e motilidade progressiva de 10 a $15 \%$ para os a base de amida e de $27 \%$ e $8 \%$, respectivamente, para os a base de glicerol. Segundo Alvarenga et al. (2005) e Oliveira et al. (2013) diluentes contendo amidas em sua formula, como o BotuCrio ${ }^{\circledR}$, fornecem melhores resultados na preservação do sêmen de equinos do que os diluidores contendo alta concentração ou 
somente glicerol, provavelmente devido à melhor permeabilidade das aminas na célula espermática e, portanto, a menores injúrias espermáticas (Alvarenga \& Papa 2011).

A porcentagem média de anormalidades morfológicas maiores, menores e totais foram significativamente menores que os valores limites recomendados pelo CBRA (2013), indicando que o sêmen dos animais poderia ser comercializado. A porcentagem de anormalidades do acrossoma foi inferior a de Mangalarga Marchador (Pugliesi et al. 2012) e as percentagens médias de defeitos totais, maiores e menores significativamente inferiores as de Quarto de Milha e Mangalarga Marchador (Costa et al. 2014) e Pônei (Araújo \& Araújo 2010) e à percentagem média de defeitos totais da raça pantaneira (Ribas 2006).

A integridade funcional da membrana plasmática do espermatozoide é fundamental para que ocorra a capacitação espermática, reação acrossomal, fusão do espermatozoide com ovócito (Maffili et al. 2003, Siqueira et al. 2007) e para a manutenção e sobrevivência da célula espermática no genital feminino (Squires et al. 1999). Para avaliação da integridade funcional da membrana plasmática do espermatozoide Jeyendran et al. (1984) propuseram o uso do teste de estresse hiposmótico (HOST) que avalia a capacidade do espermatozoide de reagir em um meio hiposmótico quando este possui a membrana plasmática funcionalmente ativa (Furst 2006). Neste estudo observou-se aumento no percentual das amostras avaliadas imediatamente após a colheita e imediatamente após a descongelação, contradizendo Oliveira (2011) que verificou queda acentuada no percentual entre as amostras avaliadas imediatamente após a colheita e após a refrigeração. De modo geral, verificou-se uma baixa resposta do sêmen caprino ao teste hiposmótico $(14,21 \pm 1,12 \%)$. A resposta ao HOST foi superior a de jumentos (Canisso et al. 2008) e inferior a de Quarto de Milha (Costa et al. 2014) e Mangalarga Marchador (Costa et al. 2014, Pugliesi et al 2012). Os resultados sugeriram que o BotuCrio ${ }^{\circledR}$ manteve a funcionalidade da membra plasmática dos espermatozoides dos animais avaliados, provavelmente por conservar sua integridade durante a criopreservação.

A integridade estrutural da membrana plasmática, avaliada pela coloração supravital, demonstrou que o diluente utilizado na criopreservação do sêmen promoveu bons resultados na raça Nordestina. A porcentagem de células viáveis foi semelhante a observada por Pugliesi et al. (2012) e superior ao de Furst (2006); Oliveira (2010) e Flores et al. (2008). Portanto, os valores observados indicaram que o processo de congelamento não promoveu danos à integridade estrutural da membrana plasmática dos espermatozoides destes garanhões da raça Nordestina, demonstrando a superior qualidade desta membrana em relação à de Mangalarga Marchador, jumento Pêga e raças europeias.

Neste estudo a percentagem de espermatozoides com membrana íntegra detectada pela coloração supravital foi apenas $19,57 \%$ superior à detectada pelo HOST. Estes resultados opuseram-se aos de Brito et al. (2003), que verificaram uma proporção superestimada de espermatozoides com membrana íntegra detectados pela coloração supravital em comparação ao HOST. Segundo estes autores isto pode ocorrer porque a coloração supravital avalia apenas os danos físicos à membrana, enquanto o HOST avalia também a atividade bioquímica, demonstrando que nem sempre um espermatozoide com integridade física tem integridade funcional.

Espermatozoides com membranas viáveis avaliados pelo SYBR-14 apresentaram fluorescência verde nítida, facilmente identificada quando contrastada com os espermatozoides mortos corados em vermelho pelo iodeto de propídio. Portanto, o uso combinado das duas sondas pode ser vantajoso na identificação de espermatozoides com membrana integra. A porcentagem de membrana íntegra avaliada pelo SYBR-14 foi superior à descrita em Quarto de Milha e Mangalarga Marchador (Costa et al. 2014). Observou-se maior nível $(\mathrm{P}<0,05)$ de detecção da integridade de membrana plasmática dos espermatozoides com a SYBR-14 em comparação com HOST, concordando com Pereira et al. (2012). Portanto, as sondas de epifluorescência podem ser usadas na avaliação de sêmen de cavalo Nordestino, com destaque para SYBR-14 que foi mais sensível que iodeto de propídio.

0 teste de ligação do espermatozoide à membrana perivitelina (MPV) da gema de ovo permite verificar a capacidade fecundante da célula espermática e, indiretamente, os eventos bioquímicos relacionados à fertilização (capacitação e reação acrossomal) (Amorim 2008). Por isso é considerado um valioso teste na análise da eficácia do processo de criopreservação do sêmen (Barbato et al. 1998). 0 número médio de espermatozoides ligados à MPV após a descongelação em garanhões da raça Nordestina foi semelhante ao descrito em caprinos (Dias et al. 2013) e em suínos da raça Piau utilizando-se o meio Beltsville thawing solution (BTS) (Pinho et al. 2012) e superior ao de garanhões (Khlifaoui et al. 2003). Estes resultados indicaram que a concentração espermática, assim como o meio de incubação utilizado, não interferiram na capacidade de adesão de espermatozoides à membrana perivitelina da gema do ovo em animais da raça.

0 teste TTR indicou que a manutenção do sêmen equino em contato com o diluente de congelamento até $10 \mathrm{ou}$ 30 minutos após o resfriamento a $5^{\circ} \mathrm{C}$ não interferiu na capacidade de movimentação e velocidade de progressão da célula espermática. Costa et al. (2014) não observaram diferenças significativas $(\mathrm{P}>0,05)$ aos 60 minutos de incubação, todavia a partir do tempo 120 minutos houveram diferenças $(\mathrm{P}<0,05)$ na motilidade espermática. Pugliesi et al. (2012) não observaram diferenças $(P>0,05)$ quanto à motilidade progressiva durante o TTR, nos tempos de 0 , 20, 40, 60 e 90 minutos em Mangalarga Marchador, contudo observaram perda de $18,4 \%$ da motilidade progressiva do sêmen descongelado ao tempo 0 do TTR em comparação ao sêmen fresco $(67,7 \%$ vs $49,3 \%)$, enquanto que Furst (2006) observou perdas de $28,6 \%$ no T0 e ao final do TTR (90 minutos) este valor não ultrapassou 50,0\%. A diminuição na integridade funcional da membrana plasmática do espermatozoide após o TTR parece ocorrer devido aos danos causados pelo processo de criopreservação nas estruturas e organelas envolvidas na movimentação espermática (Watson 1995).

A alta correlação positiva observada entre a motilidade total e a motilidade progressiva era esperada e concordou com Oliveira (2010). Por outro lado, a ausência de correla- 
ção significativa entre estas e o HOST discordou de outros autores (Melo 1999, Canisso 2008). A ausência de correlação significativa entre espermatozoides reativos ao HOST e as demais características seminais contrapôs-se ao descrito por Melo \& Henry (1999) e Melo et al. (2005) e a ausência de correlação significativa entre motilidade espermática e a capacidade de ligação dos espermatozoides à membrana perivitelina da gema de ovo ao observado por Dias et al. (2013).

\section{CONCLUSÕES}

Os resultados demonstram que a membrana plasmática de espermatozoides, de garanhões da raça Nordestina mantem sua integridade morfológica, funcional e estrutural após a criopreservação melhor que outras raças de equídeos.

0 uso combinado de diferentes testes de avaliação das injúrias espermáticas é fundamental para obtenção de informações confiáveis e precisas, sendo os resultados apresentados um parâmetro para estudos futuros nesta raça.

Agradecimentos.- À FACEPE, pela bolsa de estudos, e pelo financiamento a esta pesquisa (APQ-1072-5.04/12). À empresa BOTUPHARMA pela doação dos diluentes utilizados (BotuSêmen ${ }^{\circledR}$ e BotuCrio ${ }^{\circledR}$ ). Ao CPSENS/ FINEP por toda infraestrutura e suporte para a realização da pesquisa. À Associação Equestre e de Preservação do Cavalo Nordestino pela concessão do uso dos animais.

\section{REFERÊNCIAS}

ABCCN 1987. Regulamento do Registro Genealógico do Cavalo Nordestino. Associação Brasileira dos Criadores do Cavalo Nordestino, Recife. 33p.

Aidar N.B. 2013. Criopreservação de sêmen equino. Monografia de Graduação, Faculdade de Medicina Veterinária, Universidade de Brasília, Brasília, DF. 51p.

Almeida J.L. 2006. Efeito de diferentes concentrações de plasma seminal na criopreservação do sêmen equino. Dissertação de Mestrado em Ciências Agrárias, Faculdade de Agronomia e Medicina Veterinária, Universidade de Brasília, Brasília, DF. 90p.

Alvarenga M.A. \& Papa F.O. 2011. Principais avanços no processamento e aplicação do sêmen congelado de equinos. Spermova Suppl.1:7-10.

Alvarenga M.P., Papa F.O., Landim-Alvarenga F.C. \& Medeiros A.S.L. 2005. Amides as cryoprotectants for freezing stallion semen: a review. Anim. Reprod. Sci. 89(1/4):105-113.

Alvarenga M.A., Leão K.M., Papa F.O., Landim-Alvarenga F.C., Medeiros A.S. L. \& Gomes G.M. 2004. The use of alternative cryoprotectors for freezing stallion semen. Havemeyer Foundation Monograph Series 12:74-76.

Amorim E.A.M. 2008. Alteração da membrana espermática de suínos, bovinos e equinos na qualidade do sêmen. Tese de Doutorado em Zootecnia, Universidade Federal de Viçosa, Viçosa, MG. 174p.

Araújo A.M.S. \& Araújo S.A.C. 2010. Alterações morfológicas encontradas nas células espermáticas de garanhões Pônei. Revta Cient. Eletron. Med. Vet. 3(15), julho 2010. Disponível em <http://faef. revista.inf.br/imagens_arquivos/arquivos_destaque/ZWtYtpN6RuBhs4X_2013-6-25-15-36-32.pdf> Acessado em 20 nov. 2014.

Arruda R.P., Ball B.A., Gravance C.G. \& Liu I.K.M. 2003. Determinação da integridade da membrana plasmática e acrossomal de espermatozóides de garanhões pela técnica de citometria de fluxo. Acta Scient. Vet. 31:226-227.

Arruda R.P. 2000. Avaliação dos efeitos de diluidores e crioprotetores para o espermatozóide eqüino pelo uso de microscopia de epifluorescência, citometria de fluxo, análises computadorizadas da motilidade (CASA) e da morfometria (ASMA). Tese de Livre Docência, Universidade de São Paulo, São Paulo, SP. 121p.

Aurich C. 2005. Factors affecting the plasma membrane function of cooled stored stallion spermatozoa. Anim. Reprod. Sci. 89:65-75.

Barbato G.F., Cramer P.G. \& Hammerstedt R.H. 1998. A practical sperm-egg binding assay that detects subfertiles males. Biol. Reprod. 58:686-699.
Batellier F., Vidament M., Fauquant J., Duchamp G., Arnaud G., Yvon J.M. \& Magistrini M. 2001. Advances in cooled semen technology. Anim. Reprod. Sci. 68:181-190.

Bernardi M.L. 2008. Tecnologias aplicadas no exame do ejaculado suíno para a produção de doses de sêmen de alta qualidade. Acta Scient. Vet. 36:5-16.

Brinsko S.P., Crockett E.C. \& Squires E.L. 2000. Effect of centrifugation and partial removal of seminal plasma on equine spermatozoal motility after cooling and storage. Theriogenology 54:129-136.

Brito L.F.C., Barth A.D., Bilodeau-Goeseels S., Panich P.L. \& Kastelic J.P. 2003. Comparison of methods to evaluate the plasmalemma of bovine sperm and their relationship with in vitro fertilization rate. Theriogenology 60:1539-1551.

Candeias L.M. 2010. Avaliação de diferentes protocolos de criopreservação de sêmen de garanhões da raça Mangalarga Machador. Dissertação de Mestrado em Medicina Veterinária, Universidade Federal Fluminense, Niterói, RJ. 105p.

Canisso I.F., Souza F.A., Ker P.G., Rodrigues A.L., Sena T.C. \& Carvalho G.R. 2008. Coleta de sêmen de jumentos (Equus asinus) utilizando-se éguas em estro como manequim. Ciênc. Vet. Tróp. 11(213):57-64.

Carmo M.T. \& Almeida M.T. 2006. Biotecnologias da Reprodução aplicadas na criação de equinos. Disponível em <http://www.abqm.com.br/SecaoTecnica/biotecnologias_reproducao.html> Acessado em 14 mai. 2014.

CBRA 2013. Manual para Exame Andrológico e Avaliação de Sêmen Animal. $2^{\underline{a}}$ ed. Colégio Brasileiro de Reprodução Animal, Belo Horizonte, MG. 45p.

Celeghini E.C.C. 2005. Efeitos da criopreservação do sêmen bovino sobre as membranas plasmática, acrossomal e mitocondrial e estrutura da cromatina dos espermatozóides utilizando sondas fluorescentes. Tese de Doutorado em Reprodução Animal, Universidade de São Paulo, São Paulo, SP. 186p.

Chapon F.P. 2011. Teste hiposmótico. Monografia de Graduação, Faculdade de Veterinária, Universidade Federal do Rio Grande do Sul, Porto Alegre, RS. 28p.

CNA 2006. Estudo do complexo agronegócio do cavalo. Disponível em <http://www.cepea.esalq.usp.br/pdf/cavalo_resumo.pdf> Acessado em 15 mai. 2014.

Corcini C.D., Silva B.E., Brizolara R.M.R., Gheller S.M.M., Varela Junior A.S., Bongalhardo D.C. \& Lucia Jr T. 2012. Concentração de lactato de cálcio e tempo de incubação sobre a capacidade de adesão e penetração de espermatozoides suínos na membrana perivitelina do ovo da galinha. Ciênc. Rural 42:142-146.

Corcini C.D. 2010. Estudo de testes in vitro para predição de fertilidade de machos mamíferos. Tese de Doutorado, Universidade Federal de Pelotas, Pelotas, RS. 94p.

Costa D.N.M., Silva D.A.M., Boakari W.L., Ferreira S.B., Branco M.A.C. \& Souza J.A.T. 2014. Eficiência dos diluidores Tris e BotuCrio ${ }^{\circledR}$ sobre os parâmetros seminais de garanhões das raças Quarto de Milha e Mangalarga Marchador. Ciênc. Anim. Bras. 15(3):322-329.

Costa H.E., Filho H.C. \& Ferreira L. 2001. Exterior e Treinamento do Cavalo. Imprensa Universitária da Universidade Federal Rural de Pernambuco, Recife, PE. 169p.

Costa N., Lopes Do Vale L.J. \& Leite G.U. 1974. Estudo da preservação do cavalo nordestino. Departamento de Produção Animal, Recife, PE. 36p.

Csermak Júnior A.C. 2011. Uso de sondas fluorescentes e do ensaio de ligação do espermatozoide do cão (Canis lúpus familiaris) à membrana perivetina do ovo de galinha (Gallus gallus) como método para predição da capacidade fertilizante do sêmen. Tese de Doutorado em Medicina Veterinária, Universidade Federal de Viçosa, Viçosa, MG. 71p.

Dias J.C.O., Santos M.C.R., Penitente Filho J.M., Oliveira G.D., Mendes V.R.A. \& Mancio A.B. 2013. Teste de ligação do espermatozoide à membrana perivitelina da gema de ovo após adição de ringer lactato, citrato de sódio 2,92 \% e solução tris no sêmen caprino descongelado. Acta Vet. Bras. 7(4):300-305.

Dott H.M. \& Foster G.C. 1972. A technique for studying the morphology of mammalian spermatozoa which are eosinophilic in a differential live/ dead stain. J. Reprod. Fertil. 29:443-445. 
Enciclopédia s/d. Cavalo Nordestino. Disponível em <http://www.agrov. com/animais/equinos/nordestino.htm> Acesso em 15 mai. 2014.

Flores E., Taberner E., Ribera M.M., Pena A., Rigau T., Miro J. \& Rodriguez-Gil J.E. 2008. Effects of freeznig/thawing on motile sperm subpopulations of boar and donkey ejaculates. Theriogenology 70:936-945.

Furst R. 2006. Efeito de diferentes tempos de equilíbrio, taxas de congelamento e concentrações espermáticas na fertilidade do sêmen equino. Tese de Doutorado em Zootecnia, Universidade Federal de Viçosa, Viçosa, MG. 96p.

Gadea J. 2005. Sperm factors related to in vitro and in vivo porcine fertility. Theriogenology 24:431-444.

Garcia A.R. 2005. O uso de sondas fluorescentes na avaliação morfofuncional de espermatozoides bovinos. Revta Ciênc. Agrár., Belém, 43(Supl.):junho. Disponível em <http://ainfo.cnptia.embrapa.br/digital/bitstream/item/93719/1/Id47462.pdf> Acessado em 15 mai. 2014.

Garner D.L., Johnson L.A., Yue S.T., Roth B.L. \& Haugland R.P. 1994. Dual DNA staining assessment of bovine sperm viability using SYBR-14 and Propidium Iodide. J. Androl. 15(6):620-629.

IBGE 2010. Banco de Dados Agregados. Disponível em <http://www.sidra. ibge.gov.br/bda/tabela/listabl.asp? $\mathrm{z}=\mathrm{t} \& \mathrm{c}=73>$ Acesso em 14 mai. 2014.

Jeyendran R.S., Van der Ver H.H., Perez-Pelaez M., Crabo B.G. \& Zaneveld L.J. 1984. Development of fan assay the functional integrity of the human sperm membrane and its relationship to other semen characteristics. J. Reprod. Fertil. 70:219-228.

Kavak A., Lundeheim N., Aidnik M. \& Einarsson S. 2004. Sperm morphology in Estonian and Tori breed stallions. Acta Vet. Scand. 45:11-18.

Kenney R.M., Hurtgen J.P. \& Pierson R.W. 1983. Manual for Clinical Fertility Evaluation of the Stallion. Society for Theriogenology, Hastings, USA. (Apud Terraciano 2008)

Ker P.G. 2010. Fertilidade do sêmen congelado de jumento da raça Pêga em éguas inseminadas antes e após a detecção da ovulação. Dissertação de Mestrado em Zootecnia, Universidade Federal de Viçosa, Viçosa, MG. 81p.

Khlifaoui M., Battut I., Jean M., Bruyas J.F., Thorin C. \& Tainturier D. 2003. Assessment of fertilizing ability of frozen-thawed stallion semen in glutamine extender. J. Anim. Vet. Adv. 2(12):686-692.

Linden L.S.V.D. 2012. A avaliação da integridade da membrana se baseia no fato de que a membrana plasmática íntegra pode prevenir a entrada do corante no citoplasma celular. Dissertação de Mestrado em Medicina Animal, Universidade Federal do Rio Grande do Sul, Porto Alegre, RS. 61p.

Loomis P.R. \& Graham J.K. 2008. Commercial semen freezing: individual male variation in cryosurvival and the response of stallion sperm to customized freezing protocols. Anim. Reprod. Sci. 105:119-28.

Maffili V.V., Torres C.A.A., Fonseca J.F., Prosperi C.P., Santos A.D.F. \& Borges A.M. 2003. Uso de diferentes tempos de incubação no teste hiposmótico em sêmen caprino. Revta Bras. Reprod. Anim. 27(4):631-635.

Medeiros A.S.L., Gomes G.M., Carmo M.T., Papa F.O. \& Alvarenga M.A. 2002. Cryopreservation of stallion semen using different amides. Theriogenology 58:273-276.

Melo J.B. 2011. Caracterização zoométrica do remanescente da raça equina Nordestina nos Estados de Pernambuco e Piauí. Tese de Doutorado Integrado em Zootecnia, Universidade Federal Rural de Pernambuco, Recife, PE. 118p.

Melo M.I.V., Henry M. \& Beker A.R.C.L. 2005. Teste hiposmótico para avaliação da viabilidade do sêmen eqüino resfriado com diferentes diluidores. Arq. Bras. Med. Vet. Zootec. 57(6):757-763.

Melo M.I.V. \& Henry M. 1999. Teste hiposmótico na avaliação de sêmen equino. Arq. Bras. Med. Vet. Zootec. 51:71-78.

Melo M.I.V. 1999. Teste hiposmótico na avaliação do sêmen equino. Tese de Doutorado em Veterinária, Universidade Federal de Minas Gerais, Belo Horizonte, MG. 67p.

Moraes E.A., Graham J.K., Torres C.A., Meyers M. \& Spizziri B. 2010. Delivering cholesterol or cholestanol to bull sperm membranes improves cryosurvival. Anim. Reprod. Sci. 118(2/4):148-154.

Mortimer S.T. \& Maxwell W.M.C. 2004. Effect of medium on the kinematics of frozen-thawed ram spermatozoa. Reproduction 127:285-291.
Mugnier S., Dell'Aquila M.E., Pelaez J., Douet C., Ambruosi B., Santis T., Lacalandra G.M., Lebos C., Sizaret P.-Y., Delaleu B., Monget P., Mermillod P., Magistrini M., Meyers S.A. \& Goudet G. 2009. New insights into the mechanisms of fertilization: comparison of the fertilization steps, composition, and structure of the zona pellucida between horses and pigs. Biol. Reprod. 81:856-870.

Neild D.M., Chaves M.G., Flores M., Miragaya M.H., Gonzalez E. \& Aguero A. 2000. The HOS test and its relationship to fertility in the stallion. Andrologia 32:351-355.

Oliveira R.A., Rubins M.I.B. \& Silva C.A.M. 2013. Índice de prenhez com sêmen congelado de garanhões da raça crioula usando glicerol ou dimetilformamida como crioprotetores. Ciênc. Anim. Bras. 14(4):488-494.

Oliveira R.A. 2011. Antioxidantes na viabilidade do sêmen equino congelado e resfriado. Tese de Doutorado em Ciência Animal, Universidade Federal de Goiás, Goiânia, GO. 100p.

Oliveira R.R. 2010. Efeito in vitro da incorporação de colesterol à membrana plasmática de espermatozoide de jumentos (Equus asinus) da raça Pêga. Dissertação de Mestrado em Zootecnia, Universidade Federal de Minas Gerais, Belo Horizonte, MG. 109p.

Oliveira C.H. 2007. Avaliação das características do espermatozóide equino congelado submetido à inclusão e remoção do colesterol das membranas. Dissertação de Mestrado em Medicina Veterinária, Universidade Federal de Minas Gerais Belo Horizonte, MG. 85p.

Pereira K.F., Garcia A.R., Nahúm B.S. \& Kahwage P.R. 2012. Uso de sondas de epifluorescência para a avaliação de sêmen criopreservado bubalino. Seminário de Iniciação Científica da Embrapa, 16-17 de agosto. Embrapa Amazônia Oriental, Belém/PA, p.1-4.

Pickett B.W. \& Amann R.P. 1993. Cryopreservation of sêmen, p.769-789. In: Mickinnon A.O. \& Voss J.L. (Eds), Equine Reproduction. Lea and Febiger, Philadelphia. (Apud Almeida 2006)

Pinho R.O., Shiomi H.H., Lima D.M.A., Costa E.V., Santos M.C.R., Lopes P.S., Guimarães S.E.F. \& Guimarães J.D. 2012. Teste de ligação de espermatozoides de suínos da raça Piau à membrana perivitelina da gema do ovo. Revta Bras. Reprod. Anim 36(4):245-249.

Pugliesi G., Fürst R. \& Carvalho G.R. 2012. Efeito de diferentes tempos de equilíbrio na criopreservação de sêmen de garanhões. Revta Bras. Ciênc. Vet. 19(3):172-177.

Pugliesi G. 2009. Viabilidade e fertilidade do sêmen equino resfriado a $5^{\circ} \mathrm{C}$ por 24 horas com dois diluidores. Dissertação de Mestrado em Zootecnia, Universidade Federal de Minas Gerais, Belo Horizonte, MG. 103p.

Ribas J.A.S. 2006. Influência das estações cheias e seca nas características reprodutivas e seminais de garanhões da raça pantaneira no pantanal de Poconé/MT. Tese de Doutorado em Produção Animal, Universidade Estadual do Norte Fluminense Darcy Ribeiro, Campos dos Goytacazes, RJ. 80p.

Samper J.C. \& Hankins K. 2001. Breeding mares with frozen semen in private practice. Proc. Annual Convention of the American Association of Equine Practitioners, AAEP, San Diego, p.314-318.

Siqueira J.B., Guimarães J.D., Costa E.P., Henry M., Torres C.A.A., Silva M.V.G.B. \& Silveira T.S. 2007. Relação da taxa de gestação com sêmen bovino congelado e testes de avaliação espermática in vitro. Revta Bras. Zootec. 36:387-395.

Squires E.L., Pickett B.W., Graham D.K., Vanderwall P.M. \& Bruemmer J.E. 1999. Cooled and frozen stallion semen. Anim. Reprod. Biotechnol. Lab. 9:3-36.

Terraciano P.B. 2008. Criopreservação de espermatozoide equino comparando duas curvas de congelamento combinadas com diluentes comerciais: uma análise laboratorial. Dissertação de Mestrado em Ciências Veterinárias, Universidade Federal do Rio Grande do Sul, Porto Alegre, RS. 54p.

Torres A.P. \& Jardim W.R. 1992. Criação do Cavalo e de Outros Equinos. $3^{a}$ ed. Nobel, São Paulo.

Varner D.D., Blanchard T.L., Love C.L., Garcia M.C. \& Kenney R.M. 1988. Effects of cooling rate and storage temperature on equine spermatozoal motility parameters. Theriogenology 29(5):1043-1054. (Apud Oliveira 2007)

Watson P.F. 1995. Recent developments and concepts in the cryopreservation of spermatozoa and the assessment of their post thawing function. J. Reprod. Fertil. 7:871-891. (Apud Pugliesi et al. 2012) 